\title{
Tangence
}

\section{Vie et aventures du féminisme postmoderne d'après Irmtraud Morgner}

\section{Barbara Havercroft}

Numéro 47, mars 1995

Écritures au féminin : le genre marqué

URI : https://id.erudit.org/iderudit/025848ar

DOI : https://doi.org/10.7202/025848ar

Aller au sommaire du numéro

Éditeur(s)

Tangence

ISSN

0226-9554 (imprimé)

1710-0305 (numérique)

Découvrir la revue

Citer cet article

Havercroft, B. (1995). Vie et aventures du féminisme postmoderne d'après Irmtraud Morgner. Tangence, (47), 21-33. https://doi.org/10.7202/025848ar d'utilisation que vous pouvez consulter en ligne.

https://apropos.erudit.org/fr/usagers/politique-dutilisation/ 


\section{Vie et aventures du féminisme postmoderne d'après Irmtraud Morgner ${ }^{1}$}

\section{Barbara Havercroft, UQAM}

En dépit des nombreuses publications récentes portant sur le phénomène postmoderne et nonobstant la multitude de théories et de pratiques féministes postmodernes, il est étonnant, voire irritant, de constater la rareté des ouvrages consacrés à ces dernières et en particulier à la littérature postmoderne au féminin ${ }^{2}$. La présence de cette absence, si j'ose dire, me semble d'autant plus ironique que les théoriciens du postmodernisme insistent sur la valorisation des femmes et des autres groupes "marginalisés", pour leur accorder enfin des voix. La condition postmoderne estelle un syndrome surtout patriarcal qui incarne et réaffirme, dans ses nombreuses études et analyses métatextuelles, une des hiérarchies qu'elle prétend dissoudre, c'est-à-dire celle qui existe entre les hommes et les femmes?

Comme dans le cas de plusieurs hiérarchies fondées sur une opposition binaire, celle-ci est sexuée. Et c'est précisément sur ce

1 Cet article fait partie du projet -Glissements du gender dans le récit postmoderne au féminin•, subventionné par le FCAR, que je tiens à remercier de son soutien financier.

2 Tandis qu'il existe un bon nombre d'études écrites par des femmes portant sur la rencontre problématique entre le féminisme et le postmodernisme, la place accordée aux pratiques féministes postmodernes dans les ouvrages sur le postmodernisme écrits ou dirigés par des hommes est mince. Voir, par exemple, Matei Calinescu et Douwe Fokkema (dir.), Exploring Posimodernism, Amsterdam/Philadelphie, John Benjamins, 1990; Thomas Docherty (dir.), Postmodemism: A Reader, New York, Columbia University Press, 1993; Andreas Huyssen et Klaus R Scherpe, Postmoderne: Zeichen eines kulturellen Wandels, Reinbek bei Hamburg, Rowohlt Taschenbuchverlag, 1986; Hal Foster (dir.), The Anti-Aesthetic: Essays on Postmodern Culture, Seattle, Bay Press, 1983; Brian McHale, Postmodernist Fiction, Londres/New York, Methuen, 1987 et Constructing Posimodemism, Londres, Routledge, 1992; Ihab Hassan, The Postmodern Tum, Ohio, Ohio State University Press, 1987. Sur la littérature postmoderne au féminin, voir, entre autres, Patricia Waugh, Feminine Fictions: Revisiting the Postmodem, London, Routledge, 1989; Louise Dupré, Stratégies du vertige, Montréal, Remue-ménage, 1989; et Sally Robinson, Engendering the Subject: Gender and Self-Representation in Contemporary Women's Fiction, Albany, SUNY Press, 1991. 
terrain, celui du genre ${ }^{3}$ et du sexe, que certaines auteures postmodernes ont réagi à la condition postmoderne de leur propre exclusion, qui est, d'ailleurs, loin d'être postmoderne, si nous optons pour le sens diachronique du terme "postmoderne". Pour offrir un remède et un supplément à cette condition récurrente, j'examinerai ici une manifestation particulière de la rencontre épineuse entre féminisme et postmodernisme, à savoir la remise en question de normes et de formes rigides relatives au genre, par le biais de la dissolution de l'opposition binaire entre le masculin et le féminin, telle que l'on la retrouve dans un nombre important de textes littéraires postmodernes écrits par des femmes. Cette déconstruction, qui se manifeste dans plusieurs textes provenant de contextes linguistiques, sociaux, culturels et politiques divers, peut prendre des formes très variées ${ }^{4}$. Quelle que soit la nature de ces glissements génériques, ils accentuent le fait que le genre est une construction sociale, culturelle, discursive et politique. Comme le souligne Butler, "la terreur et l'angoisse Ique l'on ressent lorsque l'on] quitte un genre prescrit [...] met en évidence les contraintes sociales de l'interprétation du genre"s. Sans aboutir à des simples jeux linguistiques dépourvus de force politique, ces textes "chorégraphiques" ${ }^{\circ}$ soulignent toutes les injustices inhé-

3 Dans cet article, mon usage du terme - genre •, ainsi que des autres termes qui en sont dérivés (•générique:, etc.) correspond au sens du mot anglais gender.

4 ll existe, par exemple, le recyclage, dans une perspective féministe, de la figure de l'androgyne (dans The Female Man de Joanna Russ), des personnages souffrant d'une amnésie générique (voir In Transit de Brigid Brophy), ceux qui ne possèdent pas de genre identifiable (dans Sphinx d'Anne Garréta et Written on the Body de Jeanette Winterson) et ceux qui sont transsexuels ou sujets d'une métamorphose sexuelle (dans Auto-expérimentation: rapport annexe de Christa Wolf, Le sexe des étoiles de Monique Proulx, The Passion of New Eue d'Angela Carter et dans tous les récits du recueil Blitz aus beiterm Himmel, dịigé par Edith Anderson). S'ajoutent à cette liste la réécriture féministe de certains récits ou figures bibliques de nature masculine (dans Les images de Louise Bouchard, Oranges Are Not The Only Fruit de Winterson et certaines sections du roman Vie et aventures de la Trobairitz Béatrice d'Irmtraud Morgner) ainsi que le réaménagement au féminin des contes de fées traditionnels dans The Bloody Chamber d'Angela Carter.

5 Judith Butler, *Variations on Sex and Gender-, Seyla Benhabib et Drucilla Cornell (dir.), Feminism as Critique, Minneapolis, University of Minnesota Press, 1987, p. 132, ma traduction.

6 Je reprends ici l'expression de Susan Suleiman dans - (Re)Writing the Body: The Politics and Poetics of Female Eroticism *, S. Suleiman (dir.), The Female Body in Western Culture, Cambridge, Massachusetts, Harvard University Press, 1985, p. 23. 
rentes à l'opposition binaire entre le féminin et le masculin, son imbrication fondamentale aux grands récits patriarcaux et l'asservissement des femmes qui en a résulté

Un tel questionnement est au cœur du volumineux roman de l'écrivaine allemande Irmtraud Morgner, Vie et aventures de la Trobairitz Béatrice d'après les témoignages de sa ménestrelle Laura $^{7}$. Engagée dans une lutte contre les contraintes patriarcales de la société socialiste, tout comme sa compatriote Christa Wolf, Morgner aborde toutefois la question de l'émancipation des femmes de façon plus directe et plus polémique ${ }^{8}$. Son roman, riche et hétérogène, comprend une multitude de textes enchâssés de toutes sortes: des documents politiques — même le texte de la nouvelle loi sur l'avortement - ; des poèmes écrits par Béatrice; des entrevues et des lettres de Morgner concernant son propre roman; des citations de divers reportages diffusés dans des journaux et à la radio; des slogans socialistes, souvent parodiés; un tout autre récit, celui de la conductrice de tramway, Laura; des intermèdes, qui sont en fait des extraits d'un roman inédit de Morgner, intitulé Rumba pour un automne; de nombreuses références littéraires et mythologiques et un récit fantastique intitulé "La Bonne Nouvelle selon Valeska en soixante-treize strophes". Ce vaste montage textuel lance un défi soutenu aux conventions réalistes, tout en subvertissant la notion de narrateur omniscient. La juxtaposition de genres littéraires différents, accompagnée d'une ironie mordante, constitue "un refus de privilégier une forme de connaissance par rapport à une autre" 9 . À la différence des récits de Monique Wittig et d'Hélène Cixous, où

7 Publié d'abord en Allemagne de l'Est en 1974 par Aufbau Verlag, Berlin et Weimar, sous le titre Leben und Abenteuer der Trobadora Beatriz nach Zeugnissen ihrer Spielfrau Laura: Roman in dreizebn Büchern und sieben Intermezzos. Toute référence au texte sera désormais indiquée par le numéro de la page entre parenthèses et sera tirée de la traduction française (par Evelyne Sinnassamy), publiée à Paris par les Éditions des fernmes en 1983.

8 Vie et aventures a été caractérisé comme - le roman le plus important de la République démocratique allemande à l'égard de la réflexion sur l'émancipation des femmes. Voir Peter Demetz, After the Fires: Recent Writing in the Germanies, Austria, and Switzerland, New York, Harcourt Brace Jovanovich, 1992 (1986), p. 79, ma traduction.

9 Biddy Martin, - Socialist Patriarchy and the Limits of Reform: A Reading of Irmtraud Morgner's Life and Adventures of Troubadora Beatriz as Cbronicled by Her Minstrel Laura., Studies in Twentietb Century Literature, vol. V, $\mathrm{n}^{\circ} 1$, 1980 , p. 67. 
l'innovation prend souvent la voie de la fragmentation, Morgner utilise une accumulation de textes différents, une superposition de nombreux textes linéaires.

En effet, le roman dans son entier est un texte enchâssé, voire un manuscrit retrouvé, ou plutôt acheté, comme le révèle Morgner dans sa préface au récit. Dans cette brève section du roman, intitulée "Préliminaires", Morgner avoue que Laura (un personnage du roman) lui a vendu les chroniques de la vie de Béatrice de Die, à condition que Morgner rende à Béatrice les honneurs funèbres. La frontière entre fiction et "réalité. ainsi brouillée par cet échange féminin initial, une narratrice anonyme raconte ensuite la longue histoire de la femme-troubadour provençale (la "trobairitz") Béatrice de Die (un vrai personnage historique, la plus célèbre des trobairitz médiévales ${ }^{10}$ ). Jugeant insupportable le sexisme de sa France médiévale, Béatrice décide de dormir jusqu'à la fin du patriarcat, un acte accompli par l'entremise de Perséphone et de Déméter, transformées ici de leur statut antérieur mythologique de victimes du pouvoir des dieux en agitatrices rebelles politiques. Se réveillant (hélas, trop tôt!) 800 ans plus tard - toujours en France, mais en 1968 - Béatrice se rend en Allemagne de l'Est (la "terre promise" qui s'avère une déception), toujours à la recherche d'une société où les femmes seraient réellement émancipées. La narration de ses nombreuses (més)aventures engage la lectrice dans une exploration ironique des implications et de l'exactitude de tout récit historique, de toute chronologie et comme nous le verrons, de tout grand récit.

Compte tenu de la longueur ( 521 pages en traduction française) et de la complexité du roman et, par conséquent, de l'impossibilité de lui rendre justice dans un bref article, je me consacrerai uniquement à l'analyse du récit susmentionné de Valeska, qui met en relief les différences entre les sexes, entre le sexe et le genre, et également la façon dont ces différences fonctionnent pour réprimer les différences à l'intérieur de chaque terme de l'opposition binaire entre le féminin et le masculin. Conçue initialement pour la publication dans le recueil Blitz aus

10 Sur la vraie Béatrice de Die et son ceuvre, consulter Meg Bogin, The Women Troubadours, London, Paddington Press, 1976 ; Jean Boutière et A.-H. Schultz, Biographies des troubadours, New York, Lennox Hill, 1972 (1950); et William D. Padden (dir.), The Voice of the Trobairitz: Perspectives on the Women Troubadours, Philadelphie, University of Pennsylvania Press, 1989. 
beiterm $\mathrm{Himmel}^{11}$, où tous les récits portent sur le changement de sexe, la "Bonne Nouvelle* de Valeska n'a finalement pas été retenue dans cet ouvrage. Mis d'abord littéralement sous rature à cause de son caractère trop explicite, lorsque Morgner a refusé de supprimer une phrase "offensante" ${ }^{12}$ (dans ce même recueil, les hommes pouvaient écrire ainsi, alors pourquoi pas elle aussi?), le récit a été ensuite recyclé et se retrouve vers la toute fin de Vie et aventures $^{13}$. Enfin, ce même texte a été réuni avec ceux de Christa Wolf et Sarah Kirsch (déjà publiés dans le recueil Blitz) dans un ouvrage ultérieur ${ }^{14}$. Son caractère flottant s'avère d'autant plus pertinent, puisque la "Bonne Nouvelle" est introduite dans Vie et aventures en tant que manuscrit retrouvé. Ayant été déclarée cliniquement morte après un accident de la circulation, Valeska survit pour subir, plus tard, une métamorphose sexuelle qu'elle relate par écrit. Valeska, devenue un homme, confie son manuscrit à Béatrice, qui le transmet à Laura trois jours avant de mourir. La "Bonne Nouvelle " constitue une mise en abyme du roman dans son ensemble, non seulement à cause de sa reprise du passage d'un manuscrit féminin d'une femme à une autre, provoqué par la mort de Béatrice, et de la présence de commentaires explicatifs "préliminaires" au début des deux textes, mais aussi à cause de la remise en question radicale de la condition féminine et des grands récits masculins qui s'y manifeste.

11 Edith Anderson (dir.), Rostock, Hinstorff Verlag, 1975.

12 Le refus d'une rature a donc mené à la mise en place d'une autre. Morgner a apparemment choqué le directeur de la maison d'édition Hinstorff par sa phrase osée: * La couverture [du lit] sentait le tabac et le poisson. (p. 482). D'après Wolfgang Emmerich, ce genre d'expression était au-delà de ce que l'on accepte comme -violation raisonnable et salubre des tabous . Cité dans Carlotta von Maltzan, - Man müsste ein Mann sein : zur Frage der weiblichen Identität in Erzählungen von Kirsch, Morgner und Wolf •, Acta Germanica, $n^{\circ} 20,1990$, p. 144 , ma traduction.

13 Ironiquement, le récit de Valeska a fini par paraître avant le recueil où il était d'abord destiné à voir le jour. La publication de Blitz, retardée maintes fois, principalement à cause de certains empèchements de la part des collaborateurs et éditeurs masculins, date de 1975, tandis que Vie et aventures a paru en 1974. L'histoire fascinante de la publication de Blitz est racontée par la directrice du recueil, Edith Anderson, dans . Genesis and Adventures of the Anthology Blitz aus beiterm Himmel., Margy Gerber (dir.), Studies in GDR Culture 4: Selected Papers from the Ninth New Hampshire Symposium on the German Democratic Republic, Lanham, Maryland, University Press of America, 1984, p. 1-14.

14 Cet ouvrage s'intitule Gescblechtertausch: Drei Geschicten über de Umwandlung der Verbälinisse, Darmstadt/Neuwied, Luchterhand, 1980. 
26

La "Bonne Nouvelle" consiste en une histoire assez simple, dotée toutefois d'une portée significative. Mariée depuis deux ans avec le scientifique Rudolf, Valeska, elle-même scientifique, supporte cet état conjugal parce qu'elle vit avec son fils Amo, et non avec son mari, grâce à une pénurie de logements. Lorsque Rudolf lui annonce un jour sa propre "bonne nouvelle", à savoir qu'il leur a trouvé un appartement commun, un processus de réflexion approfondie se déclenche chez Valeska. Sa peur de perdre son indépendance, de devenir une femme cloîtrée dans son foyer, et sa conviction croissante qu'elle est plus qu'une "collaboratrice remplaçable" (p. 478) au travail la mènent à énoncer trois fois "malgré elle [...] le souhait anormal" (p. 485): "Faudrait être un homme" (p. 481, 484, 485). Une transformation physiologique s'ensuit et Valeska, à sa grande surprise, découvre qu'elle est devenue biologiquement un homme! Après ce changement déconcertant de sexe, que Valeska considère au mieux comme un uniforme doté de privilèges ${ }^{15}$, elle continue à fonctionner mentalement et affectivement en tant que femme, voire comme un être hybride qui entretient des relations sexuelles avec des femmes et avec Rudolf. Son côté féminin est d'autant plus accentué par l'absence de changement onomastique et pronominal, suite à la métamorphose.

Composée de 73 strophes numérotées, la "Bonne Nouvelle" est en fait enchâssée par trois textes différents: un premier paragraphe à la troisième personne, où la narratrice de Vie et aventures explique comment Laura a obtenu le manuscrit de Valeska par l'entremise de Béatrice (un paragraphe qui correspond donc aux "Préliminaires" du début de Vie et aventures); ensuite, deux phrases au présent écrites à la première personne, où Valeska annonce sa "simple doctrine", qui demeure non spécifiée; et enfin, après les 73 strophes, Valeska ajoute un dernier paragraphe, un retour au "je" et au présent, où elle précise que sa doctrine consiste en la recommandation aux femmes qu'elles aient *la foi en soi * (p. 505), insistant également que *la métamorphose ainsi décrite est pragmatique" (p. 505). Le glissement des pronoms (de la première à la troisième personne, et ensuite à la première personne) et des temps des verbes est significatif. Lors de la narration de la "Bonne Nouvelle" (les 73 strophes), Valeska se

15 La notion d'uniforme extérieur est également mise en relief par la comparaison que fait Valeska entre elle-même et l'infortunée Jeanne d'Arc, dont le déguisement était pourtant - trop voyant - (p. 487). 
sert de la troisième personne pour se décrire comme une autre ${ }^{16}$, comme une non-personne, ce qui constitue la marque linguistique de la transformation sexuelle. Le choix du registre d'énonciation que Benveniste a baptisé "histoire " ${ }^{17}$ crée une distance entre le temps de l'énoncé, le moment de la métamorphose et celui de l'énonciation, de l'écriture de la "Bonne Nouvelle", ce qui signale à la fois l'étrangeté que Valeska sent envers ellemême (c'est un "je" qui se re-présente comme une "elle") ainsi que "la vérité éternelle" de la doctrine, elle-même énoncée au présent et au "je». En outre, l'emploi du registre historique instaure un écart objectif par rapport à l'événement bizarre. Ce procédé linguistique pourrait donc être une tentative soit pour rendre l'extraordinaire plus factuel et plus plausible, soit pour conférer à l'ênoncé de 73 . strophes le caractère d'un rapport scientifique, conforme à la profession de Valeska ${ }^{18}$.

Que le récit de Valeska propose beaucoup plus qu'un simple récit humoristique de style innovateur, cela se voit d'abord dans sa critique incisive de certains grands récits masculins, utilisés pour opprimer les femmes et pour légitimer le pouvoir et les privilèges masculins. Or, c'est précisément à propos de cette question que la contribution féministe à la pensée postmoderne est indispensable. Non seulement les grands récits (qu'il s'agisse de Dieu, de la Raison, de la Nature, etc.) sont-ils masculins, créés et propagés par des hommes, mais ils sont aussi leur propriété qui, à son tour, est employée justement pour conserver ce qui appartient aux hommes: leur hégémonie, leur contrôle ${ }^{19}$. Comme le

16 Margolin se sert du terme .displaced person deixis. pour désigner un tel emploi de la troisième personne, c'est-à-dire, où le narrateur utilise un pronom inapproprié pour les fins d'autoréférence. Voir Uri Margolin, * Narrative and Indexicality: A Tentative Framework , Joumal of Literary Semantics, vol. XIII, no 3, 1984, p. 198.

17 Émile Benveniste, * Les relations du temps dans le verbe français *, Problèmes de linguistique générale, tome I, Paris, Gallimard,·1966, p. 237-250.

18 Je note en passant que le conte de Christa Wolf (Auto-expérimentation : rapport annexe), où il est aussi question d'une métamorphose sexuelle, se présente comme un rapport supplémentaire au rapport de l'expérience scientifique de changement de sexe. À cet égard, voir mon article, . Féminisme, postmodernisme et texte-supplérnent •, dans Frances Fortier (dir.), : La fiction postmoderne., Tangence, n० 39, 1993, p. 51-61.

19 A l'instar de Bordo, je parle ici de la tradition occidentale de l'homme blanc. Voir Susan Bordo, "Feminism, Postmodernism, and Gender Skepticism -, Anne Herrmann et Abigail Stewart (dir.), Theorizing Feminism: Parallel 
constate Yeatman, la notion d'individualité "consiste en la possession de propriété privée " et "les femmes, les enfants et les domestiques faisaient partie de la propriété privée des individus masculins" 20 . D'où la nécessité de démanteler, de relire et de réécrire les grands récits du passé et, selon Suleiman, les * productions culturelles dominantes" 21 . Sachant que "les hommes croient aux grandes vérités plutôt dans des habits invraisemblables" (p. 505), Morgner se lance dans un tel projet de réécriture, en commençant par le grand récit peut-être le plus connu en Occident: celui de la Bible.

De prime abord, le récit de Valeska se présente en tant que "Bonne Nouvelle" (le terme allemand est Botschaft), terme qui fait évidemment référence à la révélation chrétienne des Évangiles. Cependant, le message est détourné par Morgner pour préconiser un autre type de foi, "la foi en soi "que toute femme doit posséder. Ce petit récit féminin, substitué au grand récit religieux, reprend de façon parodique plusieurs éléments de son éminent hypotexte, afin de les "dégonfler", de les délégitimer. Tout comme le Christ, Valeska est morte ("cliniquement"), mais revient - un miracle en soi - pour subir une transformation sexuelle (au lieu d'une transfiguration) et pour répandre "l'évangile" (p. 487) qui en découle. La métamorphose de Valeska est caractérisée par d'autres femmes comme un "miracle" (p. 498), où Dieu est descendu de son paradis céleste pour s'installer dans la cafetière électrique bien terrestre ${ }^{22}$. Ces références aux grands miracles chrétiens (la résurrection et la transfiguration) mises à part, Valeska, à l'instar de Jésus, étudie d'autres miracles "pour la propagation de la doctrine", en réécrivant ceux du Christ: "je peux marcher sur des cheveux, faire de la pluie, multiplier des pains, bien sûr, ça ne suffira pas" (p. 505). Les miracles seuls et les croyances dans les grandes vérités ne sont pas susceptibles de

Trends in the Humanities and Social Sciences, Boulder, Colorado, Westview Press, 1994, p. 464.

20 Anna Yeatman, -A Feminist Theory of Social Differentiation ", Linda Nicholson (dir.), Feminism/Postmodemism, New York, Routledge, 1990, p. 287, ma traduction.

21 Susan Suleiman, op. cit., p. 18.

22 Le café fait d'abord partie intégrante de la transformation, étant donné que Valeska en buvait au moment d'énoncer le souhait anormal. (p. 498). Ensuite, Valeska réussit à provoquer le même changement corporel sans le café - Dieu n'était même pas dans la cafetière, après tout. 
changer les mentalités, même si Valeska était prête à se laisser "mettre en croix * (p. 505) pour ce faire.

C'est pour cette raison que Valeska entreprend l'écriture de sa "bonne nouvelle". La création du petit récit écrit est ainsi valorisée: "pour pouvoir entrer dans l'histoire" (p. 500), les femmes, elles aussi, ont besoin de leurs textes. En empruntant le style des versets numérotés de la Bible, tous approximativement de la même longueur, Valeska les transforme en énoncés de longueurs variables (de trois mots à plus d'une page), tout en se servant de phrases incomplètes. Le récit résultant, une sorte de contredocument face à certains grands textes masculins (même Freud et Rousseau y sont convoqués) prône la nécessité d'un respect de soi et d'une foi en soi que les femmes doivent acquérir. Ce n'est qu'en réalisant cette "simple doctrine", selon laquelle les femmes ne seront plus les symboles et les victimes de l'oppression masculine, mais jouiront d'un statut politique égal à celui des hommes, que nous pourrons accéder à "l'humanisation de l'homme" (p. 503), en évitant ainsi "une autodestruction de l'humanité par des guerres" (p. 505).

Si la Bible constitue un des grands récits patriarcaux ciblés par Morgner, le système socialiste de l'Allemagne de l'Est, un autre métarécit puissant, fait l'objet d'une critique plus atténuée. Malgré la loi qui garantissait aux femmes un salaire égal à celui des hommes (pour le même travail), malgré la célèbre Familiengesetz (la loi sur la famille) "assurant" la charge partagée des enfants, il reste que très peu de femmes œuvraient dans certaines sphères "masculines (la médecine, l'enseignement universitaire, la politique, etc.), tandis qu'elles s'occupaient toujours de $80 \%$ des tâches domestiques ${ }^{23}$. Cette disparité entre l'égalité théorique des sexes et l'inégalité dans la pratique a incité plusieurs écrivaines à dénoncer la perpétuation du patriarcat à l'intérieur du socialisme allemand ${ }^{24}$. Étant donné que l'émancipation des

23 En 1970, les femmes formaient presque la moitié de la main d'ceuvre de la République démocratique allemande et $90 \%$ des fernmes entre 18 et 60 ans travaillaient. Ces statistiques concernant la condition des femmes en R.D.A. sont fournies par Gail Finney, -Imagining the Other: Sexual Transformation and Social Reality in GDR Literature., German Life and Letters, vol. XLIV, $\mathrm{n}^{\circ} 1,1990$, p. $46-47$.

24 Dans leurs écrits, Christa Wolf, Morgner, Maxie Wander, Gerti Tetnzer et Brigitte Reimann ont toutes démontré l'écart entre la garantie légale d'émancipation et les mécanismes d'oppression qui perduraient, en dépit de la 
femmes a été utilisée comme l'indicateur du degré du progrès de la société socialiste, voire comme un de ses principes de base, et que le gouvernement se servait des chiffres "encourageants" (par rapport au nombre d'ouvrières, etc.) pour légitimer le récit et le système socialistes, les petits récits de Morgner et d'autres écrivaines jouaient un rôle capital dans la délégitimation et dans la transgression des normes établies, tant au plan social que littéraire.

La critique de l'inégalité dans la sphère privée, où le travail ne possédait pas le statut d'un produit rémunéré, prend plusieurs formes dans la *Bonne Nouvellen. De nombreux personnages féminins sont affligés de surmenage par leur double tâche (le travail à l'extérieur et au foyer), citant leur "ras le bol de cette sorte d'émancipation qui permettait aux femmes de travailler comme des hommes et par-dessus le marché comme des femmes" (p. 497). L'emploi de l'asyndète dans la liste interminable des tâches quotidiennes de Lena, une poète vivant de sa plume, met en valeur la quantité de ces besognes et la vitesse constante requise pour les accomplir: "Lena passait ses jours à courir, se lever à six heures, amener l'enfant au jardin des enfants, allumer le poêle, ranger, faire des poèmes, faire des commissions [...], laver le linge, faire la cuisine [...]" (p: 499). Le rythme incessant d'activités délirantes, connoté par cette série de verbes à l'infinitif, le manque de pauses inscrit littéralement dans cette phrase par le peu de conjonctions utilisées et l'infime place accordée au travail professionnel (l'expression "faire des poèmes " étant entourée de verbes rattachés aux tâches domestiques) créent un contraste net avec la représentation de la situation des personnages masculins du récit. Ces derniers, "habitué[s] aux ménagères"(p. 480), soutiennent que "les commissions ne devraient pas intéresser un chercheur" (p. 486) ${ }^{25}$. La responsabilité des tâches domestiques est ainsi départagée selon l'opposition binaire entre le féminin et le masculin, où un ensemble de besognes est automatiquement assigné à certains individus détenant des caractéristiques physiologiques spécifiques. L'absurdité de cette injuste bifurcation sert à

déclaration officielle de l'égalité juridique, politique et êconomique. Voir Biddy Martin, op. cit., p. 59-60.

25 Critiquer le socialisme ainsi risque de valoriser le capitalisme occidental, mais il n'en est rien dans ce roman. Morgner propose plutôt un socialisme - humanisé -, modifié à partir d'une perspective féministe. 
révéler comment le genre se construit comme "un système de sens, au lieu d'une qualité possédée par des individus ${ }^{2}{ }^{2}$.

Cette configuration binaire rigide et stéréotypée, intimement liée au maintien des grands récits, renforce l'assujettissement des femmes, car chaque récit phallologocentrique (que cela soit la Religion, la Raison, la Nature, etc.) exige un "autre" réprimé, dont "l'histoire matérielle [...] et la perspective restent encore à écrire "27. D'après cette pensée binaire, un des deux termes est toujours subordonné à l'autre, qui instaure son autorité et ses privilèges par rapport à cette tyrannie de l'autre: "cet ordoninancement dichotomique de la réalité fonctionne comme une série d'alibis, dont tous sont désignés à assurer la maîtrise du monde par l'individu possessif "28. Non seulement ces dualismes constituent-ils la structure de l'individualité patriarcale moderne, en maintenant l'invisibilité et la soumission du terme subordonné, mais l'homogénéité fabriquée de chaque partie de l'opposition cache les multiples différences en jeu à l'intérieur de chaque terme 29 .

Dans la *Bonne Nouvelle", l'opposition binaire entre le féminin et le masculin est bien mise en place en fonction des rôles et des normes attribués aux personnages féminins et masculins. Ces normes et ces rôles sont surtout érigés à partir de qualités, privilèges, comportements, discours, clichés et attentes associés typiquement aux deux sexes. D'un côté, les personnages masculins (dont Rudolf constitue l'exemple le plus significatif) s'abstiennent de participer aux tâches domestiques; ne se mettent pas en doute; jouissent d'une liberté sexuelle plus grande que celle des femmes; se donnent "l'accolade " (p. 497); croient à l'asservissement des femmes (p. 484) ; possèdent des manières "autoritaires" (p. 484) et exhibent "une joie enfantine de propriétaire [...] convoitant des regards [...] admiratifs" (p. 495). Les personnages féminins, par contre, possèdent le sens du "devoir ménager.

26 Sally Robinson, op.cit., p. 1, ma traduction.

27 Susan Bordo, op. cit., p. 459, ma traduction. Sur la politique d'oppression instaurée paŕ les dualismes occidentaux, voir également Donna Haraway, A A Cyborg Manifesto, in Simians, Cyborgs and Women: The Reinvention of Nature, New York, Routledge, 1991, p. 177.

28 Anna Yeatman, op. cit., p. 288, ma traduction.

29 Joan Scott analyse les conséquences de cette dissimulation dans - Deconstructing Equality-Versus-Difference: or, The Uses of Poststructuralist Theory for Feminism-, Anne Herrmann et Abigail Stewart (dir.), op. cit., p. 359-369. 
(p. 481); sont d'une heureuse nature (p. 482); figurent dans le même syntagme que les maximes telles que: "Il y a plus de bonheur à donner qu'à recevoir " (p. 482); sont sujettes à la demande d'une éternelle jeunesse (p. 495) et ainsi de suite. Si cette bifurcation paraît quelque peu amplifiée, elle se range néanmoins dans les idées (et souvent, dans les pratiques!) reçues et souligne la fonction des stéréotypes, à savoir: "de reproduire des sujets idéologiques qui peuvent être facilement insérés dans des institutions existantes de gouvernement, d'économie et [...] d'identité sexuelle 30 .

Bien que la critique de l'opposition binaire entre le féminin et le masculin se trouve, dans la "Bonne Nouvelle", déjà dans sa formulation stéréotypée, c'est la métamorphose sexuelle de Valeska qui provoque le véritable déplacement de ce dualisme, pour illuminer ses tendances essentialistes et l'injustice des rôles génériques. Devenue un être hybride, Valeska combine un corps d'homme et un esprit de femme; plus tard dans le récit, elle finit par pouvoir changer de sexe à son gré. Placée au-delà de la division binaire, Valeska n'a plus besoin d'adhérer aux normes qui prescrivent ou interdisent certains comportements - au contraire, elle réussit à les mettre en évidence, tout en les transgressant, comme autant de privilèges qui doivent appartenir aux femmes aussi bien qu'aux hommes. En tant qu'homme biologique, Valeska entretient des relations sexuelles avec plusieurs femmes différentes. Caractériser ces relations selon les catégories établies s'avère problématique: s'agit-il de relations hétérosexuelles ou homosexuelles? Et sa relation avec Rudolf s'est-elle donc transformée en relation entre hommes gais ? ${ }^{31}$ Ou vaut-il mieux caractériser Valeska en tant que bisexuelle? Toutes contrariétés confondues, la réponse importe peu. La pluralité hétérogène, la liberté d'action et la défamiliarisation (dans le sens des Formalistes russes) du contenu des rôles génériques servent tous à démontrer que, d'une part, la construction de l'identité est une question de pouvoir et que, d'autre part, le soi est un processus déterminé

30 Craig Owens, - The Medusa Effect •, Scott Bryson, Barbara Kruger et al. (dir.), Beyond Recognition: Representation, Power, and Culture, Berkeley, University of California Press, 1992, p. 194, ma traduction.

31 Cette possibilité semble en quelque sorte atténuée, car Valeska adopte son ancien corps de femme pour faire l'amour avec Rudolf, d'où la notion d'une hétérosexualité nécessaire qui me semble se construire parfois derrière les hêtérogénéités du récit. 
par un conditionnement complexe en termes de rôles mis en place par ceux qui détiennent le pouvoir de le faire.

Par sa forme hétéroclite, sa critique des grands récits masculins, sa dissolution de l'opposition binaire entre le féminin et le masculin pour en exposer sa nature fabriquée et abusive, ainsi que par sa réécriture de mythes et de textes patriarcaux, le roman de Morgner affirme la possibilité féminine de célébrer l'impropre, l'hétérogène, l'ex-centrique, sans pour autant tomber dans le piège postmoderne d'une simple jouissance formelle dénuée de force politique. Loin de constituer un seul "relativisme anomique ${ }^{32}$, ces aventures nous montrent que l'abandon des théories générales et universelles au profit de la diversité sociale et culturelle n'équivaut pas nécessairement à une absence d'agentivité (agency). En effet, comme les autres récits postmodernes au féminin mettant en valeur des glissements génériques, Vie et aventures évite la création textuelle d'un sujet féminin essentialiste qui nierait les différences entre les femmes.

32 J'emprunte cette expression à Anna Yeatman, op. cit., p. 292. 